\title{
Réparation ou régénération du squelette, avec des biomatériaux, comment, pourquoi ?
}

\author{
Pierre Weiss \\ INSERM / Université de Nantes, Laboratoire d'ingénierie ostéo-articulaire et dentaire (LIOAD) UMRS 791, \\ 1 place Alexis Ricordeau, 44042 Nantes Cedex 1, France ; UFR d'Odontologie de Nantes, Université de Nantes, \\ 1 place Alexis Ricordeau, 44042 Nantes Cedex 1, France
}

La médecine régénératrice est une thématique relativement récente du point de vue de sa structuration et de sa visibilité. Le vieillissement des populations avec l'objectif de «vivre bien», le plus longtemps possible, tend à vouloir substituer le concept de réparation grâce à un dispositif médical par la régénération ad intégrum du tissu lésé. En effet un dispositif médical dont l'objectif est de réparer une fonction, comme par exemple une prothèse de hanche, vieillit lui aussi. Surtout, il ne s'adapte pas aux modifications du vivant. L'interface se dégrade et la fonction disparaît. La régénération d'un tissu ou d'un organe fait appel à la médecine régénératrice dont le périmètre peut être défini à la transplantation, les biomatériaux de substitution et les nanovecteurs, la thérapie cellulaire, la thérapie génique et l'ingénierie tissulaire.

Les matériaux de substitution osseux sont des supports à l'envahissement cellulaire osseux et sont à l'interface de la réparation et de la régénération. En effet lors de leur implantation, l'effet réparation est faible et ils nécessitent souvent des dispositifs médiaux complémentaires à type d'ostéosynthèse. Puis les cellules se différentient en cellules osseuses et minéralisent les espaces avant le remaniement osseux et du biomatériau, permettant la substitution. Deux grandes classes de matériaux de substitution osseuse peuvent êtres décrits : une classe où l'effet des granules sert de support à l'adhésion cellulaire, à la différenciation des cellules et à la fabrication de matrices extracellulaires biologiques pour faire du tissu osseux ; une classe de matériaux poreux, qui présente des canaux entre des pores afin que les cellules de construction du tissu osseux puissent fabriquer la matrice osseuse et substituer le matériau pour régénérer le tissu osseux. On peut les considérer comme des matériaux de régénération à partir du moment où ils permettent de retrouver le tissu osseux ad integrum tel qu'avant la maladie ou avant l'intervention. Ces matériaux fonctionnent très bien pour de petites cavités. Ils permettent des diffusions de protéines et la migration de cellules à leur contact afin que ces cellules puissent re-fabriquer leur propre matrice extracellulaire, et donc de l'os. Les formes galéniques de substituts osseux injectables sont pour la plupart constitués de phosphate de calcium. Ils ont la même phase minérale que l'os ; ils sont non toxiques, biocompatibles, moulables ou injectables. Etant injectables, ils remplissent entièrement la cavité et permettent un contact correct entre les biomatériaux et les tissus environnants.

Ces matériaux bioactifs ne peuvent néanmoins actuellement pas régénérer de $1^{1}$ os par apposition. Seule $1^{1}$ association avec des cellules et/ou des facteurs de croissance permet $\mathrm{d}^{1}$ envisager cette régénération par ingénierie tissulaire. Il s'agit d'un sujet de recherche très important à l'heure actuelle : origines des cellules les mieux adaptées, manière de les traiter, risques, moyens de les appliquer au cabinet, quelle réglementation, etc. De nombreuses interrogations subsistent, même si l'utilisation clinique approche.

Dans les domaines de la régénération du cartilage ou du disque intervertébrale, tissus non vascularisés, les biomatériaux seuls ne peuvent que réparer une fonction lors de l'utilisation de prothèses de genoux par exemple. Il faut associer des cellules aux biomatériaux pour régénérer un tissu. C'est l'ingénieure tissulaire.

Pour réaliser ces nouveaux tissus, les biomatériaux doivent être adaptés aux types de cellules et au tissu cible. Discher et Engler ont parfaitement montré que la différenciation cellulaire était liée aux propriétés visco-élastiques d'un matériau sur la différenciation des cellules. On n'utilise pas un matériau très rigide pour fabriquer un tissu mou. Chaque type de tissu possède des propriétés visco-élastiques, qu'il convient de respecter pour pouvoir orienter des cellules vers cette différenciation. De nombreux phénomènes physicochimiques et biologiques entrent néanmoins en ligne de compte.

Pour l'os, nous choisissons des phosphates de calcium, qui sont des matériaux rigides, dont la composition est semblable à celle du tissu osseux. Pour le cartilage ou d'autres tissus mous, nous utilisons des hydrogels, qui représentent le mieux possible et de façon synthétique la matrice extracellulaire des tissus mous. On peut également associer les deux. L'équipe du LIOAD développe depuis plusieurs années des formulations d'hydrogels. Nous avons mis au point et développé un hydrogel présentant un fort potentiel en ingénierie tissulaire osseuse et articulaire. C'est un hydrogel auto-réticulant en fonction du $\mathrm{pH}$, qui est constitué d'une solution aqueuse d'hydroxypropylméthylcellulose (HPMC) sur laquelle sont greffés des groupements silanes qui permettent la formation de liaisons covalentes entre les chaînes d'HPMC.

Ce polymère est stable en solution aqueuse à un $\mathrm{pH}$ supérieur ou égal à environ 12,5. Une acidification de la solution provoque une augmentation progressive de la viscosité et la formation d'un hydrogel.

Pour le cartilage différents types d'hydrogels sont utilisables mais ces biomatériaux sont fragiles et les articulations supportent des contraintes importantes. Nous avons choisi d'utiliser des micro et nano renforts comme des silicates ou des fibres de silicium. 
En ingénierie tissulaire osseuse, les CSMs peuvent être couplées à une matrice possédant des propriétés ostéoinductrices et ostéo-conductrices de part sa porosité et sa nature chimique. Ces matrice peuvent aussi être associées à des facteurs de croissance, ou de différentiation comme les VEGF ou les BMP.

L'utilisation de cellules est très réglementé et coute cher avec de nombreuses étapes de sécurisation et de contrôle. De plus cette stratégie d'utilisation des cellules souches mésenchymateuses reste moins efficace que l'utilisation de la moelle totale.

Deux hypothèses sont mises en avant actuellement :

L'hypothèse la plus récente est une réaction lymphocytaire T dirigée contre les CSM autologues lorsque elles sont implantées en nombre dans un tissus (Liu, Nature medicine 2011).

La deuxième est l'ischémie qui associe une déplétion en glucose et en oxygène des CSM. Deschepper et Petit ont clairement montré l'action majoritaire du manque de glucose sur la mortalité cellulaire mais de nombreux auteurs révèlent également la baisse du niveau d'oxygène dès $200 \mu \mathrm{m}$ dans un biomatériaux. Sous $1 \%$ l'hypoxie n'est plus considérée comme physiologique. Même si il est vrai que les CSM supportent très bien l'hypoxie ce qui les maintient dans leur niveau souche, quand est-il réellement des cellules qui ont été en normoxie ou des cellules déjà en cours de différentiation ? Il est également notable que la revascularisation dans un substitut osseux est un processus lent qui progresse des berges vers le centre de la zone implantée. Les cellules au sein du biomatériau implanté peuvent elles attendre 2 à 3 semaines l'arrivée de néo vaisseaux?

D'autres stratégie comme l'utilisation de protéines ou mêmes de vecteurs pour la thérapie génique est également envisageable.

Les biomatériaux présentent une utilisation simple et répondent à des stratégies simples par rapport à la biologie ou au traitement de la biologie. Avec les biomatériaux, il est intéressant de pouvoir guider la biologie de l'hôte et donc de permettre de façon privilégiée la différenciation de cellules plus ou moins rapidement parce qu'une chimie, des propriétés physiques et des formulations permettent justement à ces cellules de s'exprimer du tissu hôte vers le biomatériau. La connaissance de ces biomatériaux est fondamentale pour développer des stratégies innovantes sur la régénération tissulaire.

Néanmoins, ceci fonctionne pour des problèmes simples. Avec le vieillissement de la population, d'autres stratégies permettront d'espérer régénérer des tissus vivant et éviter la réparation et l'utilisation de biomatériaux comme dispositifs médicaux qui vieillissent avec inévitablement des risques à l'interface de la prothèse si elle ne se fracture pas.

\section{Références}

Turczyn, R., Weiss, P., Lapkowski, M. \& Daculsi, G. J Biomater Sci Polym Ed 11, 217-23 (2000).; Bourges, X., Weiss, P., Coudreuse, A., Daculsi, G. \& Legeay, G. Biopolymers 63, 232-8. (2002).

Bourges X, Weiss P, Coudreuse A, (null), Legeay G. General properties of silated hydroxyethylcellulose for potential biomedical applications. 2002;63(4):232-8.

Liu Y, Wang L, Kikuiri T, Akiyama K, Chen C, Xu X, Yang R, Chen W, Wang S, Shi S. Mesenchymal stem cell\&ndash;based tissue regeneration is governed by recipient T lymphocytes via IFN-\&gamma; and TNF\&alpha. Nature Medicine. Nature Publishing Group; 2011 Nov 20;:1-9.

Deschepper M, Oudina K, David B, Myrtil V, Collet C, Bensidhoum M, Logeart-Avramoglou D, Petite H. Survival and function of mesenchymal stem cells (MSCs) depend on glucose to overcome exposure to long-term, severe and continuous hypoxia. Journal of Cellular and Molecular Medicine. 2011 Jun 22;15(7):1505-14. 